\title{
Ovarian Stromal Tumors: A Rare Cause of Postmenopausal Virilization
}

\author{
May Uyking-Naranjo and Roberto Mirasol \\ Section of Endocrinology, Diabetes and Metabolism, \\ Department of Internal Medicine \\ St. Luke's Medical Center, Quezon City, Philippines
}

\begin{abstract}
We report the case of a 61-year-old Filipino woman with postmenopausal virilization presenting with androgenic alopecia, progressive hirsutism and deepening voice over 5 years due to elevated testosterone levels. Ultrasound and computed tomography imaging suggested a left adnexal mass. Histology obtained following total abdominal hysterectomy and bilateral salpingo-oophorectomy (TAHBSO) demonstrated stromal luteoma of the left ovary. On immunohistochemistry, the tumor was positive for inhibin. Postoperatively, there was note of rapid normalization of testosterone level.
\end{abstract}

Keywords: postmenopausal virilization, hyperandrogenism, ovarian tumors

\section{INTRODUCTION}

The evaluation of clinical or biochemical androgen excess in postmenopausal women is usually not considered because of the absence of menstrual changes and the ascription of changes in hair patterns to the natural ageing process. Although the condition is rare, timely clinical recognition, knowledge of related biochemical abnormalities and risks for other diseases and implementation of risk modification of androgen excess effectively reduce morbidity and mortality.

\section{CASE}

A 61-year-old Filipina, 5 years postmenopause, was referred to the Endocrinology outpatient clinic in 2011 for evaluation of hirsutism. She presented with gradually increasing and coarsening facial hair at the upper lip and chin and deepening voice over 5 years. She had no symptoms of easy bruisability, moon facies, galactorrhea, headaches or visual changes.

She has been hypertensive for more than 20 years, and has had several emergency room consults over the last 5 years due to hypertensive urgency. She was also diagnosed with diabetes mellitus and dyslipidemia in 2005. Her maintenance medications include Losartan $100 \mathrm{mg}+$ HCTZ 25 mg OD, Amlodipine 10 mg OD, Metformin 500 $\mathrm{mg}$ BID and Simvastatin $20 \mathrm{mg}$ OD. Clonidine is taken as needed. She had no known family history of hirsutism, or gynecologic and endocrine malignancies. She had no history of tobacco, alcohol, illicit drug, steroid or herbal supplement use.

Age at menarche was 12 years, with subsequent regular menses. She had no history of dysmenorrhea or

ISSN 0857-1074

Printed in the Philippines

Copyright $(C 2013$ by the JAFES

Received March 25, 2013. Accepted April 23, 2013. menorrhagia. She is multigravid [G3P2 (1112)]. G1 (1982) was delivered preterm at 7 months via caesarian section for preeclampsia. G2 was a spontaneous pregnancy loss. G3 (1988) was full term, delivered via caesarian section. She had no history of oral contraceptive pill treatment or hormonal therapy. Age at menopause was 55. She was diagnosed with diffuse adenomyosis and uterine myomas after having recurrent hypogastric pain in 2008. These were managed conservatively.

On initial examination, she was obese (weight $74 \mathrm{~kg}$, height $1.49 \mathrm{~m}$ and BMI $33 \mathrm{~kg} / \mathrm{m}^{2}$ ) with central predominance. Blood pressure ranged between 140 to 160/90 mmg Hg. Pertinent findings included male-pattern baldness, isolated acne on the face and neck and coarse hair at the upper lip and chin. There was no excess hair growth on the chest, breast, back, abdomen or inner thigh areas (Ferriman-Gallwey score 7). There was notable loss of female body contours. Heart, lung and abdominal findings were normal. Examination of the external genitalia showed normal pubic hair distribution and clitoral enlargement $(1.5 \mathrm{~cm} \times 2.5 \mathrm{~cm})$. Further gynecologic examination was otherwise normal. The rest of the physical examination was unremarkable (Figures $1 \mathrm{~A}$ to $\mathrm{C}$ ).

Out-patient workup showed elevated serum total testosterone and 17-hydroxyprogesterone (17-OHP), with normal levels of dehydroepiandrosterone sulfate (DHEAS) and a negative overnight dexamethasone suppression test. Stimulated 17-OHP ruled out non-classical congenital adrenal hyperplasia $(<10 \mathrm{ng} / \mathrm{mL})$ (Table 1$)$.

The patient was admitted in May 2012 due to episodic epigastric pain and jaundice, for which she underwent abdominal computerized tomography (CT) scan. This revealed acute pancreatitis, choledocholithiases

Corresponding author: May L. Uyking-Naranjo, MD

St. Luke's Medical Center

279 E. Rodriguez Sr. Boulevard,

Quezon City, Philippines 1112

E-mail: may22uyking@yahoo.com 
and an incidental finding of an enhancing structure measuring $5.1 \mathrm{~cm} \times 2.8 \mathrm{~cm} \times 4.0 \mathrm{~cm}$ on the left adnexa (Figure 2). She underwent endoscopic retrograde cholagiopancreatography (ERCP), sphincterotomy and basket extraction. She was discharged improved after 3 days.

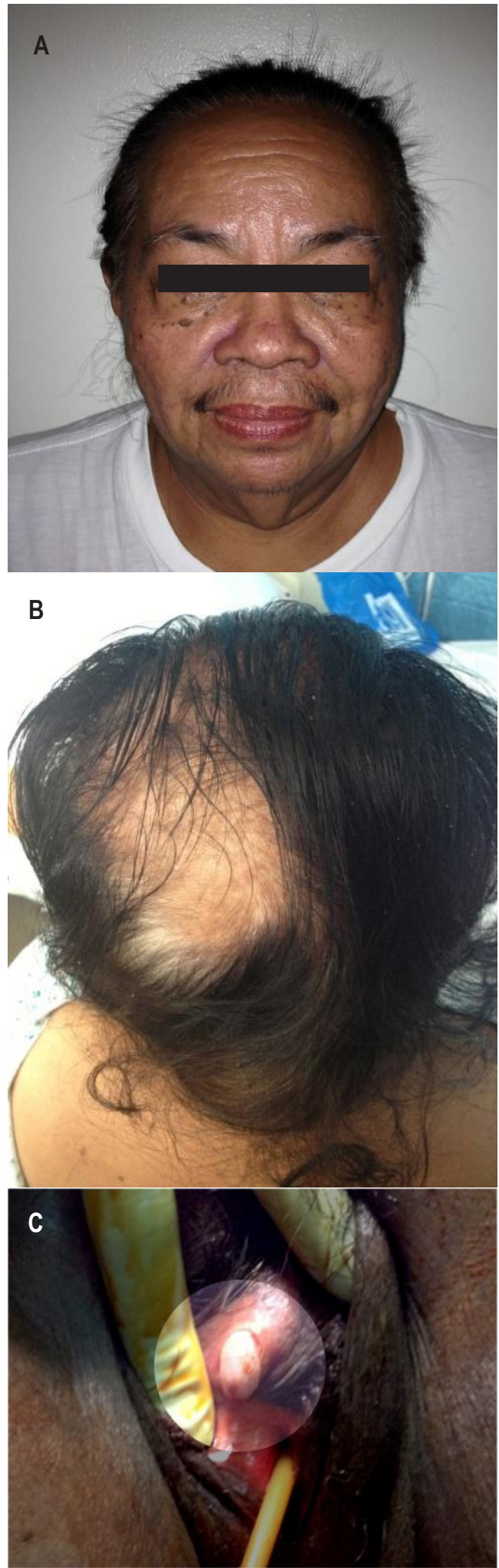

Figure 1. Examination revealed masculinized features, with coarse terminal hair at the upper lip and chin $(A)$; androgenic alopecia (B); and clitoromegaly (C).
Table 1. Pre and post-operative hormonal assays

\begin{tabular}{lccc}
\multicolumn{1}{c}{ Hormone } & $\begin{array}{c}\text { Pre- } \\
\text { operative }\end{array}$ & $\begin{array}{c}\text { Post- } \\
\text { operative }\end{array}$ & $\begin{array}{c}\text { Reference } \\
\text { range }\end{array}$ \\
\hline Total testosterone $(\mathrm{ng} / \mathrm{dL})$ & 529 & $<20.00$ & $15-74$ \\
17-OHP $(\mathrm{ng} / \mathrm{mL})$ & 7.98 & 0.86 & $0.13-0.51$ \\
Stimulated $17-\mathrm{OHP}(\mathrm{ng} / \mathrm{mL})$ & 8.87 & & \\
DHEA-S $(\mu \mathrm{g} / \mathrm{dL})$ & 104 & & $35-430$ \\
Serum cortisol $(\mu \mathrm{g} / \mathrm{dL})$ & 0.94 & \\
after $1 \mathrm{mg}$ DST & \multicolumn{3}{|l}{} \\
\hline 17-OHP, hydroxyprogesterone; DHEA-S, dehydroepiandrosterone \\
sulfate; DST, dexamethasone suppression test
\end{tabular}

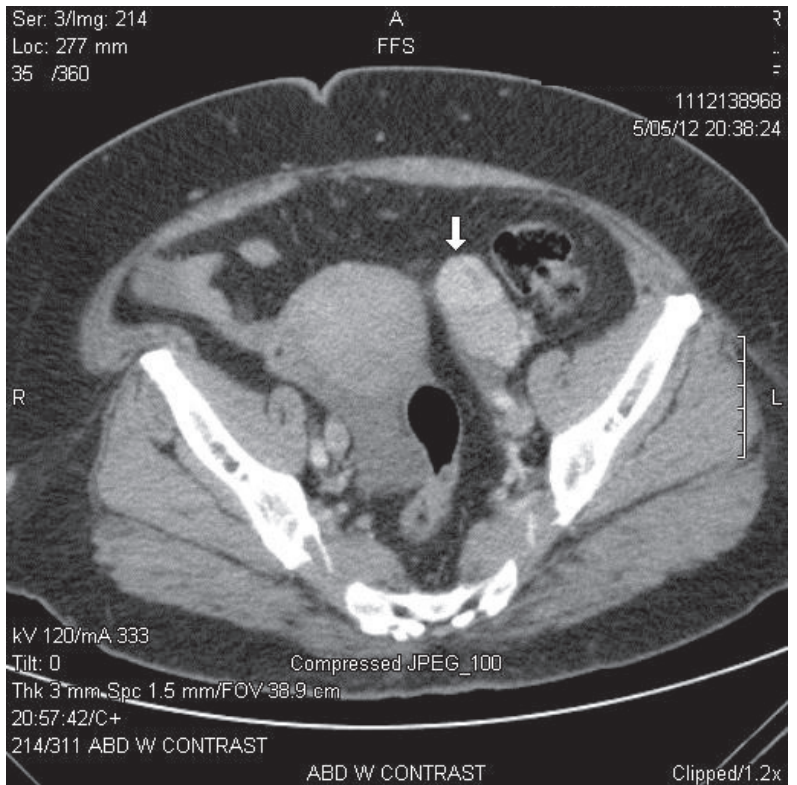

Figure 2. Contrast-enhanced CT-scan of the whole abdomen showing an enhancing structure on the left adnexa (arrow).

Further workup with transvaginal ultrasonography showed a thickened, hyperechoic endometrium $(0.72 \mathrm{~cm})$ at the upper half of the uterus, with minimal intracavitary fluid. The lower endometrium measured $0.37 \mathrm{~cm}$. There were 7 discrete uterine myomas, and a solid hypoechoic mass measuring $3.9 \mathrm{~cm} \times 3.2 \mathrm{~cm} \times 2.3 \mathrm{~cm}$ in the left adnexa with rich intratumoral vascularity. The consideration was a pedunculated subserous myoma, but an ovarian new growth could not be totally ruled out (Figures $3 \mathrm{~A}$ and B).

Differential diagnosis includes: Obesity-induced hyperandrogenism, hyperthecosis, nonclassical congenital adrenal hyperplasia and adrenal neoplasm may present with similar clinical findings. The adrenal glands were normal on imaging by CT scan, which ruled out the possibility of a testosterone-producing adrenal adenoma.

The patient underwent TAHBSO after extensive preoperative investigation. Intraoperative findings revealed a $16 \mathrm{~g}$ left adnexal complex mass. On gross examination, the left ovary had smooth, cream tan external surface and a lobulated, yellow-orange solid cut surface measuring $4 \mathrm{~cm} \times 2 \mathrm{~cm} \times 1 \mathrm{~cm}$ (Figure 4). The left fallopian tube was unremarkable $(3 \mathrm{~cm}$ in length by $0.5 \mathrm{~cm}$ in diameter). Further examination of the excised specimen showed intramural leiomyomas, adenomyosis, 
endometrial polyp, weakly proliferative endometrium, and chronic cervicitis with Nabothian cysts. The right ovary showed germinal inclusion cysts. The right fallopian tube was unremarkable.
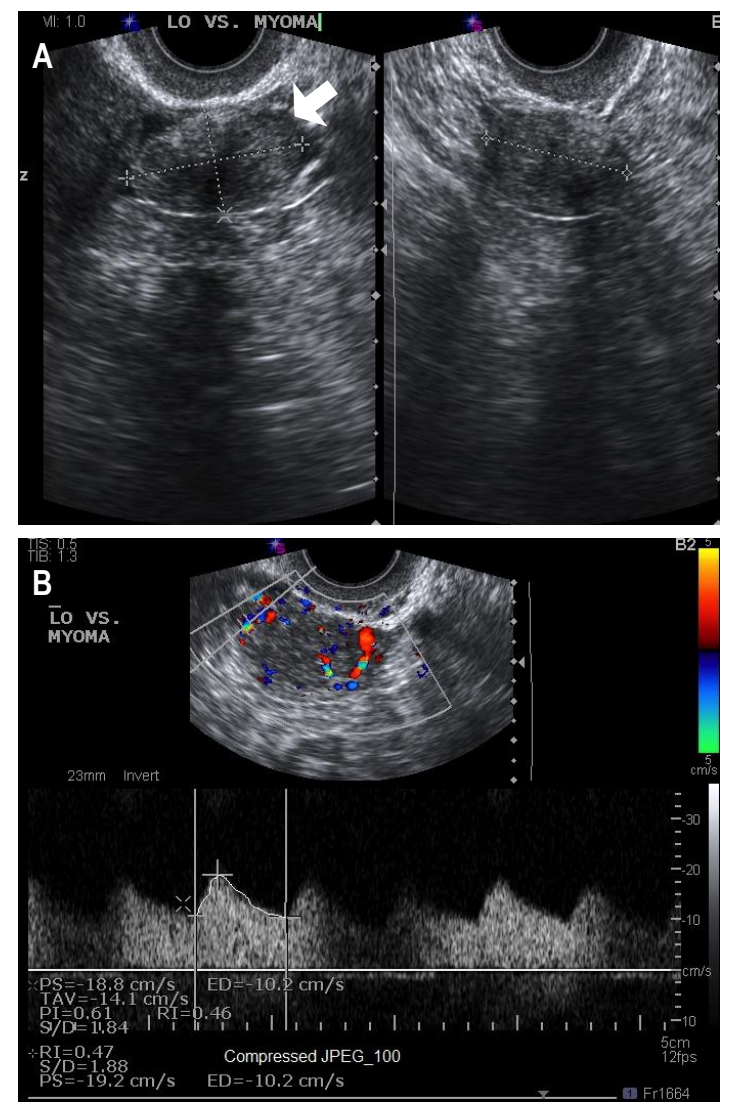

Figure 3. Transvaginal ultrasonogram showing a solid hypoechoic structure (arrow) within the left adnexa (A). Doppler examination of the left adnexal mass showed rich intratumoral vascularity on color flow mapping with high resistance wave pattern, suggestive of a benign nature (B).

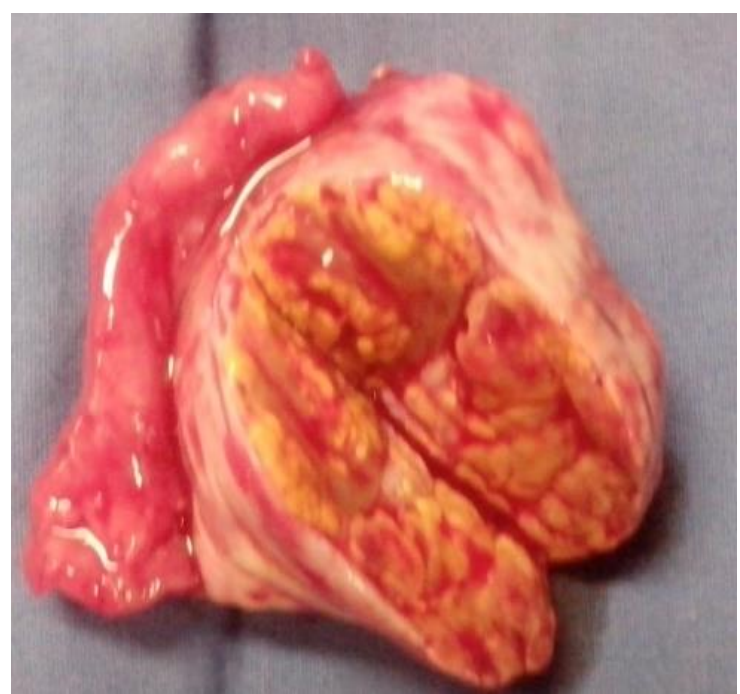

Figure 4. Gross examination left adnexal complex. The left ovary had a smooth, cream tan external surface and a lobulated, yellow-orange cut surface. Attached is the fimbriated and unremarkable left fallopian tube.
Histopathologic examination revealed ovarian stromal luteoma of the left ovary. On immunohistochemistry, the tumor was stained positive for inhibin (Figure $5 \mathrm{~A}$ to $\mathrm{C}$ ).

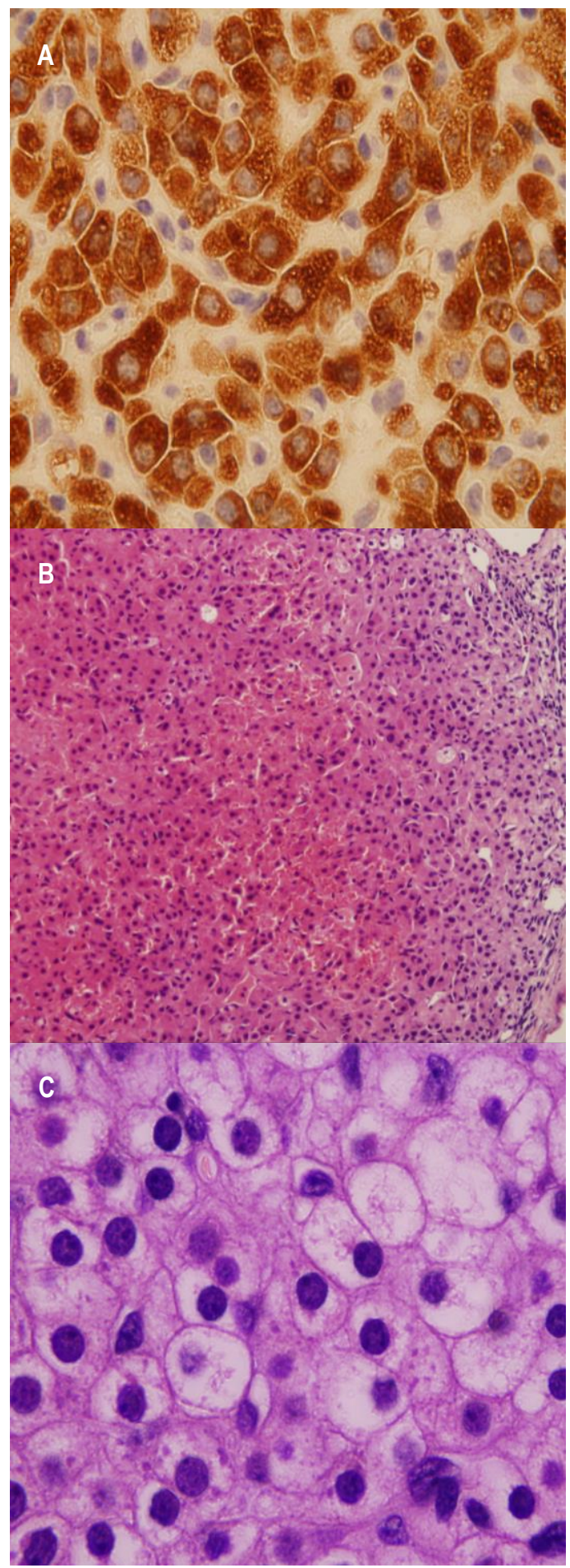

Figure 5. Microscopic examination of excised left ovary showing sheets of polygonal cells with eosinophilic to pale and granular cytoplasm, small round nuclei and prominent nucleoli. No mitoses nor crystalloid of Reinke crystals were noted (A) (H\&E, x1000). Surrounding irregularly shaped degenerative spaces were filled with hemorrhage (B) (H\&E, x400). Diffuse and strong cytoplasmic inhibin immunoreactivity were noted (C) (Inhibin, x1000). 
Postoperatively, the patient experienced rapid normalization of the elevated testosterone level and 17OHP level (Table 1). Three months after surgery, the patient remains well and asymptomatic, with no progression of alopecia and hirsutism. Blood pressure remains controlled with two antihypertensive medications. Blood sugars remained stable with Metformin 1 gram daily.

\section{DISCUSSION}

Androgen-secreting ovarian tumors represent about $1 \%$ of all ovarian neoplasms. Steroid cell tumors (SCT) are among the less common variants, which account for less than $0.1 \%$ of all ovarian tumors. ${ }^{1,2}$ The SCTs are tumors composed of cells that resemble steroid hormone-secreting cells. The three major categories of SCTs are stromal luteoma, Leydig cell tumors that lack Sertoli cell or stromal component, and steroid cell tumor not otherwise specified (NOS). ${ }^{3,4}$ In a review of 105 steroid cell tumors of the ovary, stromal luteomas account for about $22 \%$ of cases. ${ }^{5}$ These benign functional neoplasms, first described by Scully in 1964, are believed to be of stromal derivation, originating from luteinized cells or their precursors, or undifferentiated spindle cells of the ovarian stroma. ${ }^{6}$ About $60 \%$ of cases present with estrogenic manifestations, and only $12 \%$ of cases are androgenic. ${ }^{1,2}$ They are usually encountered in postmenopausal women, typically during workup for abnormal bleeding or for virilizing/feminizing symptoms..$^{2,6}$ Occasionally, they may occur as unsuspected findings during surgery. ${ }^{7}$ Some reported estrogenic manifestations include endometrial hyperplasia and well-differentiated endometrioid adenocarcinoma. $^{2}$

A previously reported case of an undifferentiated NOS steroid cell tumor presented with hirsutism, amenorrhea, clitoromegaly, and temporal baldness. ${ }^{8}$ In our patient's case, hirsutism was associated with signs of virilization in the form of deepening voice, clitoromegaly, frontal baldness and increased muscularity. In androgensecreting ovarian tumors, serum testosterone levels are often high, but DHEA-S levels are low. Our patient also had grossly elevated serum testosterone but normal DHEA-S levels.

The diagnosis of these rare tumors can be problematic, especially in the case of a small ovarian tumor. These tumors are typically less than $3 \mathrm{~cm}$ in diameter, which explains poor visualization with ultrasonography and computerized tomography. ${ }^{4}$ In previous case reports, selective venous sampling have been shown to be highly effective in tumor localization. ${ }^{9}$ However, this is an invasive and operator-dependent procedure with the risk of hemorrhage. A few case reports have previously described gonadotropin-dependent stromal luteoma, but these tumors could not be localized with imaging techniques. Testosterone, FSH and LH were markedly inhibited following the administration of a $\mathrm{GnRH}$ analogue, suggesting a gonadotropin-dependent, testosterone-secreting ovarian tumor; and implying that a stromal luteoma is not autonomous but is gonadotropindependent. ${ }^{10}$ In our case, computerized tomography incidentally detected a left adnexal mass, which was not clinically palpable; and was subsequently confirmed by transvaginal ultrasonography. A different kind of luteoma can appear in pregnancy. In the Philippines, one case of a maternal pregnancy luteoma responsible for virilization of both newborn and mother was reported, which was not the case in this patient. ${ }^{12}$

Microscopically, stromal luteomas are composed of round polyhedral cells present in nests that form nodules. Crystalloids of Reinke are conspicuously absent, a distinguishing feature of stromal luteomas from Leydig cell tumors. ${ }^{2}$ In difficult cases, immunocytochemistry provides diagnostic accuracy. The most useful immunohistochemical marker for their identification is alpha-inhibin, which is positive in most neoplasms in the sex cord-stromal group. ${ }^{4}$ Stromal hyperthecosis has been found in association with stromal luteomas in the surrounding or contralateral ovary in $90 \%$ of cases, a feature not seen in our patient. ${ }^{2,12}$

In the evaluation of postmenopausal androgen excess, the history and physical examination direct the appropriate laboratory and radiologic evaluation. Testosterone and DHEA-S are the primary hormonal tests that should be measured. ${ }^{13}$ A testosterone level above $200 \mathrm{ng} / \mathrm{dL}$ or DHEA-S level more than $800 \mathrm{ng} / \mathrm{mL}$ suggest the need to evaluate for a tumor of the ovary or adrenal. In a study of 478 women (both premenopausal and post-menopausal) with signs and symptoms of hyperandrogenism, 11 had testosterone level above $250 \mathrm{ng} / \mathrm{dL}$. However, only one of these 11 had a tumor. Of the 10 women with DHEA-S level above $600 \mathrm{ng} / \mathrm{mL}$, none had an adrenal tumor. ${ }^{14}$ Several reports have also more recently confirmed that absolute levels of elevation of these steroid hormones do not clearly differentiate the etiologies. Some have suggested a 2- to 5-day low dose dexamethasone suppression test. Failure to suppress baseline elevation of testosterone or DHEA-S is thought to indicate an ovarian source. ${ }^{15}$ However, this approach has not been studied among the postmenopausal women.

Pelvic ultrasonography or magnetic resonance imaging (MRI) is useful in women with elevated testosterone levels to evaluate the ovary. The expertise of the ultrasonographer may influence detection, as most tumors are quite small. A CT or MRI of the adrenals is indicated in the evaluation of patients with high DHEA-S, or signs and symptoms and laboratory abnormalities suggestive of adrenal Cushing's syndrome. Although most patients with isolated elevation of testosterone have an ovarian source of hyperandrogenism, there are rare case reposts of testosterone-secreting adrenal ademomas. ${ }^{16}$ Thus, imaging the adrenals is useful before proceeding to ovarian surgery. 
Data is limited concerning the frequency and severity of androgen excess in the menopause. No data is available concerning long-term effects of altering androgen levels. However, high androgens adversely alter lipid profile with increase LDL, decrease in HDL and increase triglyceride levels. ${ }^{17,18}$ There have been recent associations reported between levels of advance glycation endproducts and testosterone levels in post-menopausal women, independent of insulin resistance. High testosterone and estrogen are both associated with worsening insulin resistance and can worsen hypertension and fluid retention. Recent studies have shown that high testosterone in women correlate with increased risk for breast cancer and cardiac risk. ${ }^{18,19}$ In a group of 390 postmenopausal women, 104 of these with history of irregular cycles and hyperandrogenemia had more evidence of coronary artery disease by angiogram, as well as more obesity, metabolic syndrome and diabetes. ${ }^{18,20}$ This emphasizes the need for thorough evaluation and treatment in postmenopausal women who present with hyperandrogenism. An interdisciplinary approach to management is strongly recommended.

\section{CONCLUSION}

This case highlights the importance of a thorough evaluation in postmenopausal women who present with virilization and hyperandrogenism. The physical manifestations of androgen excess also portend the serious health risks associated with this condition. The cardiometabolic consequences of hyperandrogenemia, particularly due to underlying insulin resistance, leading to diabetes, dyslipidemia and worsening hypertension, should also be evaluated and treated.

\section{Acknowledgements}

Our special thanks to Dr. Gail Domecq C. Tanawit and Dr. Rolando A. Lopez from the Department of Pathology of the St. Luke's Medical Center, Quezon City for the photomicrographs and the histologic examination results.

\section{References}

1. Tavassoli FA, Devilee PE, eds. World Health Organization classification of tumours. Pathology and genetics of tumours of the breast and female genital organs. Lyon: IARC Press, 2003.

2. Hayes MC, Scully RE. Stromal luteoma of the ovary: A clinicopathological analysis of 25 cases. Int J Gynecol Pathol 1987;6(4):313-21.

3. Young RH, Scully RE. Sex cord-stromal, steroid cell, and other ovarian tumours with endocrine, paraendocrine and paraneoplastic manifestations. In: Kurman RJ, ed. Blaustein's pathology of the female genital tract, $5^{\text {th }}$ ed. New York: Springer-Verlag, 2002.

4. Roth LM. Recent advances in the pathology and classification of ovarian sex cord-stromal tumors. Int J Gynecol Pathol 2006;25(3):199215.

5. Paraskevas M, Scully RE. Hilus cell tumor of the ovary. A clinicopathological analysis of 12 Reinke crystal-positive and nine crystal-negative cases. Int J Gynecol Pathol 1989;8(4):299-310.

6. Scully RE. Stromal luteoma of the ovary. A distinctive type of lipoidcell tumor. Cancer 1964;17:769-78.

7. Dhingra KK, Sharma $\mathrm{P}$, Khurana $\mathrm{N}$ et al. Ovarian stromal luteoma in a patient with endometrial carcinoma. J Obstet Gynaecol Res 2008;34(2):283-5.
8. Reedy MB, Richards WE, Ueland F et al. Ovarian steroid cell tumors, not otherwise specified: A case report and literature review. Gynecol Oncol 1999;75(2):293-7.

9. Stephens JW, Katz JR, McDermott $\mathrm{N}$ et al. An unusual steroidproducing ovarian tumour: Case report. Hum Reprod 2002;17(6):1468-71.

10. Chico A, García JL, Matías-Guiu X et al. A gonadotrophin dependent stromal luteoma: A rare cause of postmenopausal virilization. Clin Endocrinol (Oxf) 1995;43(5):645-9.

11. Animas RM. Luteoma of pregnancy. Philippine Journal of Reproductive Endocrinology and Infertility. 2009;6. http://psrei.org/?page_id=406

12. Case records of the Massachusetts General Hospital. Weekly clinicopathological exercises. Case 24-1993. N Engl Med 1993;328(24):1770-6.

13. Martin KA, Chang RJ, Ehrmann DA et al. Evaluation and treatment of hirsutism in premenopausal women: An Endocrine Society clinical practice guideline. J Clin Endocrinol Metab 2008;93(4):1105-20.

14. Waggoner W, Boots LR, Azziz R. Total testosterone and DHEAS levels as predictors of androgen-secreting neoplasms: A populational study. Gynecol Endocrinol 1999;139(6):394-400.

15. Kaltsas GA, Isidori AM, Kola BP et al. The value of the low-dose dexamethasone suppression test in the differential diagnosis of hyperandrogenism in women. J Clin Endocrinol Metab 2003;88(6):2634-43.

16. Cordera F, Grant C, van Heerden J et al. Androgen-secreting adrenal tumors. Surgery 2003;134(6):874-80.

17. Wild RA, Carmina E, Diamanti-Kandarakis E et al. Assessment of cardiovascular risk and prevention of cardiovascular disease in women with the polycystic ovary syndrome: A consensus statement by the Androgen Excess and Polycystic Ovary Syndrome (AE-PCOS) Society. J Clin Endocrinol Metab 2010;95(5):2038-49.

18. Rothman MS, Wierman ME. How should postmenopausal androgen excess be evaluated? Clin Endocrinol (Oxf) 2011;75(2):160-4.

19. Dorgan JF, Stanczyk FZ, Kahle LL et al. Prospective case-control study of premenopausal serum estradiol and testosterone levels and breast cancer risk. Breast Cancer Res 2010;12(6):R98.

20. Shaw LJ, Bairey Merz CN, Azziz R et al. Postmenopausal women with a history of irregular menses and elevated androgen measurements at high risk for worsening cardiovascular event-free survival: Results from the National Institutes of Health-National Heart, Lung, and Blood Institute sponsored Women's Ischemia Syndrome Evaluation. J Clin Endocrinol Metab 2008;93(4):1276-84. 